\title{
Time-Temperature Interaction in the Induction of Pleomorphism in Trichophyton mentagrophytes
}

\author{
By DEANA T. KLEIN \\ Department of Microbiology and Immunology, Albert Einstein College of \\ Medicine, New York 61, New York, U.S.A.
}

(Received 22 July 1963)

\begin{abstract}
SUMMARY
The frequency of appearance of mutant pleomorphic patches on the surface growth of granular strains of Trichophyton mentagrophytes was markedly increased by incubation at $36^{\circ}$ as compared to the number seen at $26^{\circ}$. A minimum of 8-9 days of incubation at the higher temperature was required to induce the higher frequency of pleomorphic mutants and, as the number of days at $36^{\circ}$ increased, the number and relative size of the pleomorphic patches also increased. Typical mutants could be isolated whether the granular culture was incubated for 8-9 or 14 days at $36^{\circ}$. Visual scoring was relatively accurate since most of those patches visually scored as pleomorphic were found on isolation to be typical or atypical mutants. Three phenotypic characters were used here to define pleomorphism: type of surface mycelium, pigmentation on reverse side of colony, and effect of methionine. These characters could change independently of one another. Thus, pleomorphism may be the result of alterations in more than one closely linked chromosome-borne locus.
\end{abstract}

\section{INTRODUCTION}

Sabouraud (1910) described an unusual mutation, pleomorphism, manifested as tufts or patches of fluffy white aerial mycelium on the appressed powdery surface growth of dermatophytic fungi. He found that a medium with a high concentration of carbohydrate ( $5 \%$ glucose, maltose or mannitol) and incubation at $30-37^{\circ}$ favoured the development of the pleomorphic patch in all species of Trichophyton studied. Sabouraud emphasized the critical nature of these two parameters of the phenomenon, since no patches developed when he used a medium lacking sugar or an incubation temperature below $30^{\circ}$. Robbins (1950) and Bistis $(1959,1960)$ also reported the occurrence of pleomorphic patches when cultures of $T$. mentagrophytes were grown at $35^{\circ}$ on a medium containing $5 \%$ glucose. Bistis (1959) noted the absence of pleomorphic mutants in cultures of $T$. mentagrophytes at $25^{\circ}$. To define the phenomenon of pleomorphism and to classify presumptive pleomorphic isolates as many stable morphological and biochemical criteria of pleomorphism as possible should be used. Thus, the original definition of pleomorphism in Trichophyton advanced by Sabouraud (1910) and extended by Klein (1963) will be used here: a typical granular culture has a granular (grainy) surface growth, is pigmented on the reverse side of the colony, and is methionine sensitive. A typical pleomorphic mutant exhibits a fluffy white surface growth, is unpigmented on the reverse, and is methionine insensitive. $T$. mentagrophytes forms few macroconidia and many 
microconidia while the pleomorphic mutant loses the ability to form macroconidia but continues to produce microconidia, sometimes in large numbers (Sabouraud, 1910; Bistis, 1959; Klein, unpublished). Since cultural conditions also influence the number of both macro- and micro-conidia produced (Bistis, 1959; Chin \& Knight, 1957), sporulation is not included in this working definition of pleomorphism in $T$. mentagrophytes.

In delimiting the phenomenon of pleomorphism it is important to determine the environmental parameters under which pleomorphic mutants are formed and to determine whether the induction and growth of these mutants can be placed under precise environmental control. The work reported describes the relation of time and temperature of incubation to appearance of the pleomorphic mutation and advances the conclusion that frequency of mutation is modified by temperature.

\section{METHODS}

Organisms. Granular strains of Trichophyton mentagrophytes tested were M12-4, 4 a 5, T 689 N, T 16-4, T 16-141, T-9, T 17-3 (from the New York Botanical Garden, NYBG); X-1, X-7, X-26, X-28, X-31, X-44, X-205, X-404 (from Communicable Disease Center, CDC, Atlanta, Georgia, U.S.A.); 9129, 9633 (from American Type Culture Collection, ATCC, Washington, D.C., U.S.A.). The typical pleomorphic strain of T. mentagrophytes used was T16-12 (New York Botanical Garden).

Culture media. The basal medium contained (g. or ml./l.) $\mathrm{MgSO}_{4} .7 \mathrm{H}_{2} \mathrm{O}, 0.5$; $\mathrm{KH}_{2} \mathrm{PO}_{4}, 1.5 ; \mathrm{K}_{2} \mathrm{HPO}_{4}, 0.15$; trace element solution, 0.5; thiamine, 0.01 ; agar, 15. The nitrogen source was usually $0.2 \%(w / v)$ Difco Neopeptone. In certain experiments vitamin-free acid-hydrolysed casein (Nutritional Biochemicals Corp., Cleveland, Ohio), $20 \mathrm{ml}$./l., or a mixture of $19 \mathrm{~L}$-amino acids (California Corporation for Biochemical Research, Los Angeles, California, U.S.A.), each at the same concentration as found in hydrolysed casein, and including tryptophan, was substituted for the Neopeptone (Klein, 1963). The carbon source was usually $2 \%(w / v)$ glucose; it was replaced in certain experiments by $4 \%(\mathrm{w} / \mathrm{v})$ glucose or mannitol or $2 \%(\mathrm{w} / \mathrm{v})$ soluble starch. All media were adjusted to $\mathrm{pH} 5 \cdot 7$.

Conditions of growth. Stock cultures were grown in test tubes on slopes of Neopeptone $2 \%$ glucose agar medium and stored at $5^{\circ}$. Cultures used as a source of inoculum were grown in Petri dishes on Neopeptone $2 \%$ glucose agar medium at $26^{\circ}$. Inocula for all experiments were $4 \mathrm{~mm}$. diam. discs of agar cut from the advancing edge of mycelial growth with a sterile cork borer and placed, one disc per plate, in the centre of the agar medium. Cultures were incubated at $26^{\circ}$ or $36^{\circ}$ or in a sequence of these two temperatures. A humid atmosphere was maintained and the agar medium did not dry out during the extended incubation periods. Two or three plates were used per variable and each experiment was repeated at least once.

Isolation from pleomorphic patches. At the end of most experiments isolations were made from patches visually scored as pleomorphs. As small an amount of mycelium as possible was taken from each patch with a glass needle since isolation of too much mycelium, particularly too deeply in the patch, usually resulted in a mixed growth of pleomorph and granular. The isolates were grown on Neopeptone $2 \%$ glucose agar medium and incubated at $26^{\circ}$ for $10-14$ days when each colony was scored for type of surface growth and presence of pigmentation on the reverse 
side. Subcultures were then made from each colony to a $19 \mathrm{~L}$-amino acid $2 \%$ glucose agar medium and incubated at $26^{\circ}$ for 7-10 days at which time each was scored for methionine effect (Klein, 1963). The few isolates which proved to be mixtures of granular and pleomorphic growth were eliminated from further evaluation because their occurrence was random.

\section{RESULTS}

\section{Time-temperature interaction}

When granular strains of Trichophyton mentagrophytes were grown on Neopeptone $2 \%$ glucose (or $4 \%$ glucose or $2 \%$ soluble starch) agar medium at $26^{\circ}$ for up to 35 days pleomorphic patches were rarely seen. With strain $M$ 12-4 an average of one patch was seen per 4-5 Petri dishes; this was considered to be the spontaneous frequency. When granular strains were incubated at $36^{\circ}$ for 21 days the following results were observed. All NYBG strains showed pleomorphic patches; certain strains (M12-4, T689N, T 16-141, T 17-3) exhibited an average of 6-9 patches per plate. The carbon source did not affect the number of patches formed. The other NYBG strains showed fewer patches. The CDC strains were more variable in response; strains $X-28, X-44$ and $X-205$ showed pleomorphs (2-4 per plate), the latter two only when the carbon source was soluble starch. ATCC strain 9129 showed patches comparable in number to NYBG strain M12-4; strain 9533 showed no patches.

Granular strain M12-4 was chosen for use in the following experiments because the number of pleomorphs which occurred on the surface growth was consistently high. The length of time of incubation at $36^{\circ}$ required to induce the development of pleomorphic patches was determined. Neopeptone $4 \%$ glucose agar plates were inoculated with the granular culture and kept at $36^{\circ}$. Control plates were incubated at $26^{\circ}$. Daily, up to 14 days, two or three plates were transferred from $36^{\circ}$ to $26^{\circ}$. Thus, samples of the fungus were grown from 1 to 14 days at $36^{\circ}$, with subsequent incubation at $26^{\circ}$ (Table 1 ; Pl. 1, figs. 1-4). No pleomorphs were seen on plates incubated at $26^{\circ}$ (Pl. 1, fig. 2); about 8-9 days of continuous incubation at $36^{\circ}$ were required to induce development of pleomorphs. As the time at $36^{\circ}$ increased, the size and number of individual patches increased (Pl. 1, figs. 1, 3, 4). The order of presentation of the higher temperature was not a factor in the final response, since the typical number and average size of pleomorphs was seen at 21 days even when the 8-9 days at $36^{\circ}$ came at the end rather than at the beginning (Table 1 ) of the 14-day incubation period.

A number of modifications of the medium, including addition of $0.5 \%(w / v)$ Difco yeast extract, $2 \%$ glucose or $4 \%$ mannitol instead of $4 \%$ glucose, hydrolysed casein or the mixture of $19 \mathrm{~L}$-amino acids in place of Neopeptone, did not change the pattern of response in that no patches were observed on plates at $26^{\circ}$ while 8-9 days at $36^{\circ}$ induced the development of typical pleomorphic patches. No pleomorphs developed, however, when the carbohydrate was omitted from the medium although there was still an appreciable amount of growth; this observation confirms that of Sabouraud (1910). Thus, temperature seems to be the key inducing-factor of pleomorphism in many strains of Trichophyton mentagrophytes as long as the medium is not a limiting factor. 
Table 1. Relation between number of days of incubation at $36^{\circ}$ and frequency of appearance of pleomorphic patches on the surface of granular cultures of Trichophyton mentagrophytes strain M12-4; subsequent incubation was at $26^{\circ}$

Observations were made at 14 and 21 days after inoculation. Results summarize the average of 4 experiments.

Pleomorphic patches observed

\begin{tabular}{|c|c|c|c|c|c|c|}
\hline \multirow{2}{*}{$\begin{array}{l}\text { Time of } \\
\text { incubation } \\
\text { at } 36^{\circ} \\
\text { (days) }\end{array}$} & \multicolumn{3}{|c|}{14 days } & \multicolumn{3}{|c|}{21 days } \\
\hline & Present & $\begin{array}{l}\text { Average } \\
\text { no./plate }\end{array}$ & $\begin{array}{l}\text { Size* } \\
\text { range }\end{array}$ & Present & $\begin{array}{c}\text { Average } \\
\text { no./plate }\end{array}$ & $\begin{array}{l}\text { Size* } \\
\text { range }\end{array}$ \\
\hline 0 & 0 & - & - & $\mathbf{0}$ & - & - \\
\hline $1-7$ & 0 & - & - & 0 & - & - \\
\hline 8 & \pm & $0 \cdot 1$ & $\mathbf{S}$ & \pm & $0 \cdot 3$ & $\mathbf{S}$ \\
\hline 9 & \pm & $0 \cdot 1$ & $\mathbf{S}$ & + & $0 \cdot 6$ & $\mathbf{S}$ \\
\hline 10 & \pm & 0.4 & $\mathbf{S}$ & + & $\mathbf{2 \cdot 4}$ & S-M \\
\hline $11-13$ & $\bar{t}$ & $1 \cdot 2$ & $\mathbf{S}-\mathbf{M}$ & + & $\mathbf{3 \cdot 2}$ & $\mathbf{S}-\mathbf{L}$ \\
\hline 14 & + & $1 \cdot 6$ & $\mathbf{S}-\mathbf{L}$ & + & $5 \cdot 0$ & S-L \\
\hline
\end{tabular}

Table 2. Classification of cultures isolated from pleomorphic patches which formed on the surface of granular cultures of Trichophyton mentagrophytes strain M12-4 following increasing number of days of incubation at $36^{\circ}$

Data presented summarize four experiments. The + indicates presence of pigmentation and methionine sensitivity; the 0 indicates absence of pigmentation and methionine insensitivity.

\begin{tabular}{|c|c|c|c|c|}
\hline \multirow{2}{*}{$\begin{array}{c}\text { Time of } \\
\text { incubation } \\
\text { at } 36^{\circ} \\
\text { (days) }\end{array}$} & \multirow[b]{2}{*}{$\begin{array}{l}\text { No. } \\
\text { pleomorphs } \\
\text { isolated }\end{array}$} & \multicolumn{3}{|c|}{ Classification } \\
\hline & & $\begin{array}{c}\text { Type of } \\
\text { surface growth* }\end{array}$ & Pigmentation & $\begin{array}{c}\text { Methionine } \\
\text { effect }\end{array}$ \\
\hline $1-7$ & o & & & \\
\hline 8 & $\mathbf{I}$ & Fuzzy & $\mathbf{0}$ & 0 \\
\hline 9 & $\mathbf{3}$ & $\left\{\begin{array}{l}\text { Fuzzy } \\
\text { Fluffy }(2)\end{array}\right.$ & $\begin{array}{l}\mathbf{0} \\
\mathbf{0}\end{array}$ & $0^{+}$ \\
\hline 10 & 6 & $\left\{\begin{array}{l}\text { Granular (3) } \\
\text { Fuzzy (2) } \\
\text { Fuzzy }\end{array}\right.$ & $\begin{array}{l}+ \\
0 \\
0\end{array}$ & $\begin{array}{l}+ \\
0 \\
+\end{array}$ \\
\hline 11 & 10 & $\left\{\begin{array}{l}\text { Granular (3) } \\
\text { Fuzzy (4) } \\
\text { Fuzzy } \\
\text { Fluffy (2) }\end{array}\right.$ & $\begin{array}{l}+ \\
0 \\
+ \\
0\end{array}$ & $\begin{array}{l}+ \\
0 \\
0 \\
0\end{array}$ \\
\hline 12 & 6 & $\left\{\begin{array}{l}\text { Granular } \\
\text { Fuzzy (2) } \\
\text { Fuzzy (3) }\end{array}\right.$ & $\begin{array}{l}+ \\
0 \\
+\end{array}$ & $\begin{array}{l}+ \\
0 \\
0\end{array}$ \\
\hline 13 & 15 & $\left\{\begin{array}{l}\text { Granular (2) } \\
\text { Fuzzy (4) } \\
\text { Fuzzy (3) } \\
\text { Fuzzy (2) } \\
\text { Fluffy (4) }\end{array}\right.$ & $\begin{array}{l}+ \\
0 \\
+ \\
+ \\
0\end{array}$ & $\begin{array}{l}+ \\
0 \\
0 \\
+ \\
0\end{array}$ \\
\hline 14 & 15 & $\left\{\begin{array}{l}\text { Granular (3) } \\
\text { Fuzzy (2) } \\
\text { Fuzzy (4) } \\
\text { Fluffy (6) }\end{array}\right.$ & $\begin{array}{l}+ \\
0 \\
+ \\
0\end{array}$ & $\begin{array}{l}+ \\
0 \\
0 \\
0\end{array}$ \\
\hline
\end{tabular}

* Those described as granular are identical with parental granular appearance. 


\section{Classification of isolated pleomorphic patches}

Longer incubation at $36^{\circ}$ did not result in the induction of a higher frequency of typical pleomorphs (Table 2); furthermore, typical and atypical pleomorphs were isolated from patches which formed after 9 or 14 days exposure of strain M 12-4 to $36^{\circ}$. With few exceptions, pleomorphs with atypical (fuzzy) surface growth were methionine insensitive; isolates showing typical pleomorphic surface growth (fluffy) were invariably insensitive to methionine. These observations confirm the previous report on methionine effect (Klein, 1963).

There was no correlation between type of isolated colony and appearance of the patch; most cultures scored as granular, for example, were isolated from small, flat, or fuzzy beige patches, but typical and atypical pleomorphs also came from similar small patches. There was a greater variation in appearance of the patches than in appearance of the resulting cultures.

\section{DISCUSSION}

Elevated temperatures have been shown to increase the frequencies of gene mutation (Plough, 1941), chromosomal aberration (Sax, 1937) and cytoplasmic mutation (Ogur, Ogur \& St John, 1960; Papazian, 1955). It would appear that the pleomorphic alteration in many strains of Trichophyton mentagrophytes is yet another genetic system in which temperature plays a vital role. Whether elevated temperatures increase the frequency of the pleomorphic alteration or, on the other hand, serve only to activate the growth of a pre-existing mutant cell is not known. Bistis (1959) concluded, without experimental evidence, that the mutation may occur at $\mathbf{2 5}^{\circ}$ but that the mutant cell does not grow at this temperature. The data presented in this report do not shed any light on this important question.

The site of the mutational event is also an important unsolved problem since it is not known whether the mutation occurs in the nucleus of the conidium, the nucleus of a hyphal cell, or whether it is non-nuclear. The simplest explanation, at the moment, is that the pleomorphic mutation involves the alteration of more than one chromosome-borne locus. This is supported by the data which show that alterations of the surface character of the mycelium, capacity to form pigment, and methionine sensitivity, may or may not occur simultaneously. This fact has been commented on previously (Klein, 1962). Mutants have been isolated in this study and previously (Klein, 1961) which are non-pigmented, methionine insensitive, but not truly fluffy; or which are fuzzy and non-pigmented, but are methionine sensitive; or which are fuzzy and methionine sensitive, but are pigmented. These observations argue against pleomorphism's being the result of the mutation of a pleiotropic gene (Sinnott, Dunn \& Dobzhansky, 1958), but do not preclude the possibility that typical pleomorphism in Trichophyton mentagrophytes might be the result of a chromosomal aberration.

This investigation was supported by grant E-2876 from the National Institute of Allergy and Infectious Diseases, U.S. Public Health Service. Thanks are extended to Mrs Geraldyne White for her excellent technical assistance. 


\section{REFERENCES}

Bistrs, G. N. (1959). Pleomorphism in the dermatophytes. Mycologia, 51, 440.

BIstis, G. N. (1960). Pleomorphism and growth cycles in Trichophyton mentagrophytes. Mycologia, 52, 394.

Chin, B. \& KNIGHT, S. G. (1957). Growth of Trichophyton mentagrophytes and Trichophyton rubrum in increased carbon dioxide tensions. J. gen. Microbiol. 16, 642.

KLEIN, D. T. (1961). Effect of ionizing radiation on Trichophyton. Amer. J. Bot. 48, 539.

KLEIN, D. T. (1962). Kinetics of pleomorph induction in Trichophyton. Amer. J. Bot. 49, 655.

KuEIN, D. T. (1963). Morphological response of Trichophyton mentagrophytes to methionine. J. gen. Microbiol. 31, 91.

OGUR, M., OGUR, S. \& St John, R. (1960). Temperature dependence of the spontaneous mutation rate to respiratory deficiency in Saccharomyces. Genetics, 45, 189.

Papazian, H. P. (1955). Sectoring variants in Schizophyllum. Amer. J. Bot. 42, 394.

Plough, H. H. (1941). Spontaneous mutability in Drosophila. Cold Spr. Harb. Symp. quant. Biol. 9, 127.

Robrins, W. J. (1950). Growth requirements of dermatophytes. Ann. N.Y. Acad. Sci. $50,1357$.

Sabouraud, R. (1910). Les Teignes. Paris: Masson et Cie.

SAX, K. (1937). Chromosome behaviour and nuclear development in Tradescantia. Genetics, 22, 523.

Sinnotr, E. W., Dunn, L. C. \& Dobzhansky, T. (1958). Principles of Genetics, 5th ed. New York: McGraw-Hill Book Co., Inc.

\section{EXPLANATION OF PLATE}

Figs. 1-4. Appearance of pleomorphic patches on surface growth of granular cultures of Trichophyton mentagrophytes strain M12-4 after various times at $36^{\circ}$. Medium was Neopeptone $4 \%$ glucose agar. Photographs were taken 21 days after inoculation.

Fig. 1. Fourteen days at $36^{\circ}$ followed by 7 days at $26^{\circ}$; arrow indicates the change in growth habit between $36^{\circ}$ and $26^{\circ}$.

Fig. 2. Twenty-one days at $26^{\circ}$.

Fig. 3. Ten days at $36^{\circ}$ followed by 11 days at $26^{\circ}$.

Fig. 4. Twelve days at $36^{\circ}$ followed by 9 days at $26^{\circ}$. 
Journal of General Microbiology, Vol. 34, No. 1

Plate 1
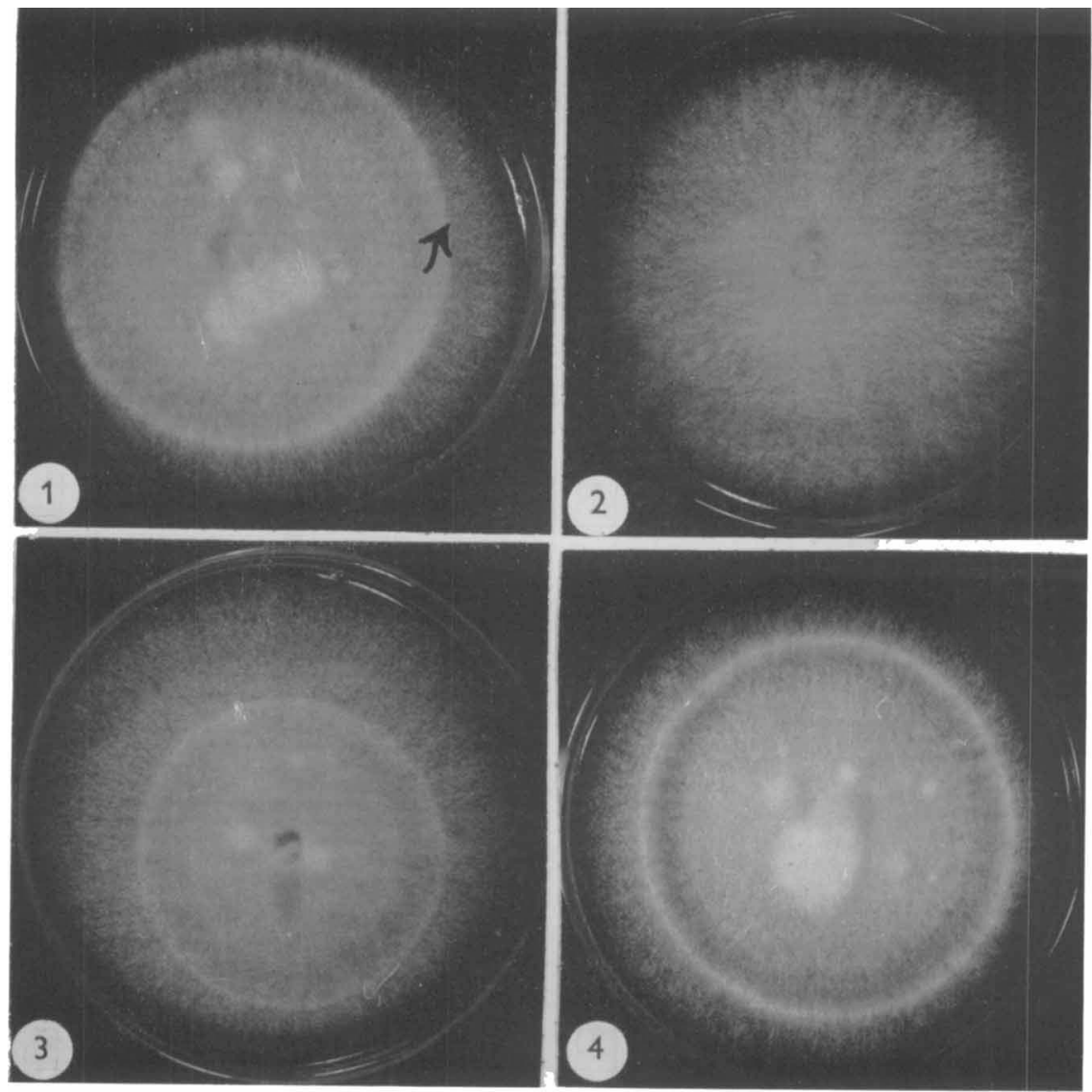

D. T. KLEIN

(Facing p. 130) 